Research Paper

\title{
Diagnostic Accuracy of a CA125-Based Biomarker Panel in Patients with Pancreatic Cancer: A Systematic Review and Meta-Analysis
}

\author{
Qingcai Meng1,2,3*, Si Shi1, 2,3*, Chen Liang1,2,3*, Jinfeng Xiang1, 2,3, Dingkong Liang1, 2, 3, Bo Zhang1, 2, 3, Yi
} Qin ${ }^{1,2,3}$, Shunrong $\mathrm{Ji}^{1,2,3}$, Wenyan $\mathrm{Xu}^{1,2,3}$, Jin $\mathrm{Xu}^{1,2,3}$, Quanxing $\mathrm{Ni}^{1,2,3}$, and Xianjun $\mathrm{Yu}^{1,2,3 凶}$

1. Department of Pancreatic Surgery, Fudan University Shanghai Cancer Center, Shanghai 200032, China;

2. Department of Oncology, Shanghai Medical College, Fudan University, Shanghai 200032, China;

3. Pancreatic Cancer Institute, Fudan University, Shanghai 200032, China.

* These authors contributed equally to this work.

$\square$ Corresponding author: Xianjun Yu, Pancreatic Cancer Institute, Fudan University; Department of Pancreatic \& Hepatobiliary Surgery, Fudan University Shanghai Cancer Center, 270 DongAn Road, Shanghai 200032, P. R. China. Tel.: +86-021-64175590 ext. 1307, Fax: +86-021-64031446. E-mail: yuxianjun@fudanpci.org

(1) Ivyspring International Publisher. This is an open access article distributed under the terms of the Creative Commons Attribution (CC BY-NC) license (https://creativecommons.org/licenses/by-nc/4.0/). See http://ivyspring.com/terms for full terms and conditions.

Received: 2016.12.24; Accepted: 2017.03.31; Published: 2017.10.09

\begin{abstract}
Background: Increasing evidence from recent studies has revealed the association of CA125 with the diagnosis of pancreatic cancer, but inconsistent findings have been reported. We aimed to evaluate the diagnostic value of a serum CA125-based diagnostic panel in predicting malignant pancreatic cancer.

Materials and Methods: We searched EMBASE, MEDLINE and Web of Science for relevant articles from inception to October 2016. Methodological quality was evaluated using the Quality Assessment of Comparative Diagnostic Accuracy Studies (QUADAS-2) checklist. The performance characteristics were pooled using random-effects models. The statistical analysis was performed using Meta-Disc 1.4 and Stata Version 12.0 software.

Results: A total of 1235 participants pooled from 8 eligible studies were included in the meta-analysis to evaluate the accuracy of tumor predictors for the diagnosis of pancreatic cancer. The pooling accuracy analysis of CA 125 alone indicated that the pooled sensitivity was 0.59 ( $95 \% \mathrm{Cl}: 0.54-0.62)$ and the specificity was $0.78(95 \% \mathrm{Cl}: 0.75-0.82)$, whereas the serum CA125-based diagnostic panel had a pooled sensitivity of $0.47(95 \% \mathrm{Cl} 0.47-0.51)$ and a specificity of 0.88 (95\% Cl 0.86-0.90). Furthermore, the AUC and Q-value of the CA125-based diagnostic panel were 0.89 and 0.82 , respectively, which indicated that the CA125-based panel is superior to CA125 or CA19-9 alone in diagnosing pancreatic cancer. No obvious publication bias was found.

Conclusions: In summary, a CA125-based diagnostic panel is better at diagnosing pancreatic cancer than a test using CA125 or CA19-9 alone. Further studies should be performed to confirm our conclusion.
\end{abstract}

Key words: CA125, pancreatic cancer, diagnosis, meta-analysis.

\section{Introduction}

Pancreatic cancer is one of the most lethal solid organ malignancies, with a 5-year survival rate of $6 \%$, and is characterized by diagnostic difficulty, distant metastasis and aggressive local invasion at an early stage [1-3]. Despite the use of advanced imaging methods and image-guided biopsy procedures in differentiating pancreatic cancer from benign pancreatic diseases, diagnostic limitations still exist [4, 5]. Furthermore, these procedures have drawbacks, as they are invasive and expensive and can only be performed in experienced centers. In the search for simple and inexpensive methods, a wide range of 
serum biomarkers including carbohydrate antigen 19-9 (CA19-9), CA125, CA50, CA724, CA242 and carcinoembryonic antigen (CEA) have been studied in connection with pancreatic cancer [6-8]. Among these, the most common and best-studied marker for pancreatic cancer is CA19-9, which is reported to have a sensitivity ranging between $68 \%$ and $92 \%[9,10]$. However, Lewis antigen-negative individuals, comprising approximately 5 to $10 \%$ of the population, exhibit little or no secretion of CA19-9, which has been the major shortfall for CA19-9 as a biomarker [11]. Furthermore, it may lack sufficient sensitivity and specificity for certain patients with specific metastases or jaundice [12-14].

As a high-molecular-weight mucin-like glycoprotein, CA125 has been reported to be a marker for epithelial cell ovarian cancer [15]. Moreover, it has been detected in colorectal adenocarcinoma, duodenal minor papilla adenocarcinoma and cholangiocarcinoma [16-18]. It has only recently been recognized that CA125 also has an important clinical value for the detection of pancreatic cancer [19-23]. In this regard, our previous studies demonstrated that CA125 is superior to CA19-9 in predicting the resectability of pancreatic cancer [24]. Furthermore, CA125 also correlates with the metastasis-associated burden, and a preoperative serum combination of CEA+/CA125+/CA19-9 > $1000 \mathrm{U} / \mathrm{mL}$ could be a useful preoperative predictor for the presence of micrometastasis in pancreatic cancer patients $[25,26]$.

Therefore, the present meta-analysis was performed to investigate the diagnostic value of CA125 and a CA125-based tumor marker panel, which could provide more reliable evidence that may help clinicians diagnose and treat pancreatic cancer.

\section{Materials and Methods}

\section{Search strategy}

A comprehensive and systematic literature review was undertaken by searching EMBASE, MEDLINE and Web of Science from inception to October 2016 using the terms 'pancreatic cancer/neoplasm/tumor/adenocarcinoma' and 'CA125/Cancer Antigen 125/CA-125' in combination with keywords, namely, 'diagnosis', 'serum' and 'biomarker'. Two reviewers (Meng QC and Shi S) independently screened the titles, abstracts and full texts of selected studies to assess eligibility. The following inclusion criteria had to be fulfilled: (a) clinical studies (cohort and case-control) of patients with pancreatic cancer; (b) studies focusing on the relationship between serum CA125, CA19-9 or CEA concentration and pancreatic cancer; (c) serum samples obtained from patients with pancreatic cancer before treatment; (d) data regarding diagnostic accuracy, such as sensitivity and specificity. The exclusion criteria were as follows: (a) published in a non-English language; (b) no full text available; (c) literature published as reviews, case reports, letters, editorials and expert opinions. Any discrepancies between the reviewers were resolved by discussion or consultation with a third reviewer (Liang C).

\section{Quality assessment}

Two reviewers (Meng QC and Shi S) independently systematically assessed the methodological quality of each study using the QUADAS-2 (Quality Assessment of Diagnostic Accuracy Studies. The checklist of the tool is as follows: (1) patient selection; (2) conduct of the index test; (3) reference standard; (4) flow and timing. The studies could be considered to be of high quality if they enrolled all consecutive, or a random sample of, eligible patients, an index test was always conducted and interpreted prior to the reference standard, and all participants underwent the same reference standard testing method [27]. The assessment results were presented as low, high or unclear risk of bias/concerns regarding applicability for each checklist topic.

\section{Data extraction}

The data were reviewed and extracted independently by two reviewers (QC Meng and Shi $S)$, and disagreements between the reviewers were resolved by a third reviewer (Liang C), using the Preferred Reporting Items for Systematic Reviews and Meta-Analyses (PRISMA) statement [28]. The following information from each article was extracted: name of first author; year of publication; country of study; number of samples; origin of samples; cut-off criteria; the number of true positive (TP), false positive $(\mathrm{FP})$, true negative (TN), and false negative (FN) results; and use of a CA125-based diagnosis panel.

\section{Statistical analysis}

The data synthesis was performed using the statistical methods described in the protocol [29]. The following summary accuracy measures with corresponding 95\% confidence intervals were obtained: sensitivity, specificity, positive likelihood ratio (PLR), negative likelihood ratio (NLR), diagnostic odds ratio (DOR). We explored the variation in the accuracy indices graphically using Forest plots and the area under the curve (AUC), a comprehensive representation of test accuracy that combines sensitivity with specificity. The heterogeneity among studies was measured using the $\mathrm{I}^{2}$ test. The Mantel-Haenszel fixed-effects model was 
applied in cases of low heterogeneity $\left(\mathrm{I}^{2}<50\right)$, and the random-effects model was applied for datasets showing obvious heterogeneity ( $\left.{ }^{2}>50\right)$ [30]. The latent publication bias was assessed by a funnel plot and Deeks' linear regression test [31]. All statistical analyses were conducted by Meta-Disc 1.4 (XI Cochrane Colloquium, Barcelona, Spain) and Stata Version 12.0 software (Stata Corporation, College Station, TX, USA).

\section{Results}

\section{Literature screening and study characteristics}

The process of literature screening and study selection is presented in Figure 1. After a preliminary online search, a total of 458 potentially relevant articles were retrieved from the EMBASE, MEDLINE and Web of Science library databases. Of these, 402 articles were excluded after a manual screening of titles, abstracts and key words because they were duplicates or irrelevant to the current analysis. After a careful review of the full texts of the remaining 56 studies, another 48 were removed in accordance with the inclusion and exclusion criteria. Finally, 8 eligible studies with 1235 participants in total were enrolled in this meta-analysis $[7,8,24,32-36]$.
The basic information and main characteristics of the eligible studies are summarized in Table 1 . The participants were enrolled in these studies were from Finland, the United States, China, Turkey, Japan or Poland. The studies dealt with a wide range of gastrointestinal diseases including pancreatic cancer, pancreatic benign tumors, pancreatitis, and other gastrointestinal diseases. Pancreatic cancer was histologically or cytologically confirmed by the pathological examination of resected specimens or by fine-needle aspiration. Patients were staged according to the TNM staging criteria for pancreatic cancer in the 7th edition of the American Joint Committee on Cancer (AJCC) Cancer Staging Manual [37]. Serum samples were collected prior to operation or chemotherapy and used for detecting the levels of tumor markers including CA125, CA19-9 and CEA by electrochemiluminescence immunoassays. The level of CA125 alone was detected in all eligible articles, and its diagnostic accuracy was evaluated, while a total of 12 groups with a CA125-based tumor panel were also studied in this meta-analysis. Four studies used the critical value of CA125 $(35 \mathrm{U} / \mathrm{mL})$ as the cut-off value, while $19.7 \mathrm{U} / \mathrm{mL}, 32 \mathrm{U} / \mathrm{mL}, 33 \mathrm{U} / \mathrm{mL}$, and $65 \mathrm{U} / \mathrm{mL}$ were used in the other studies.

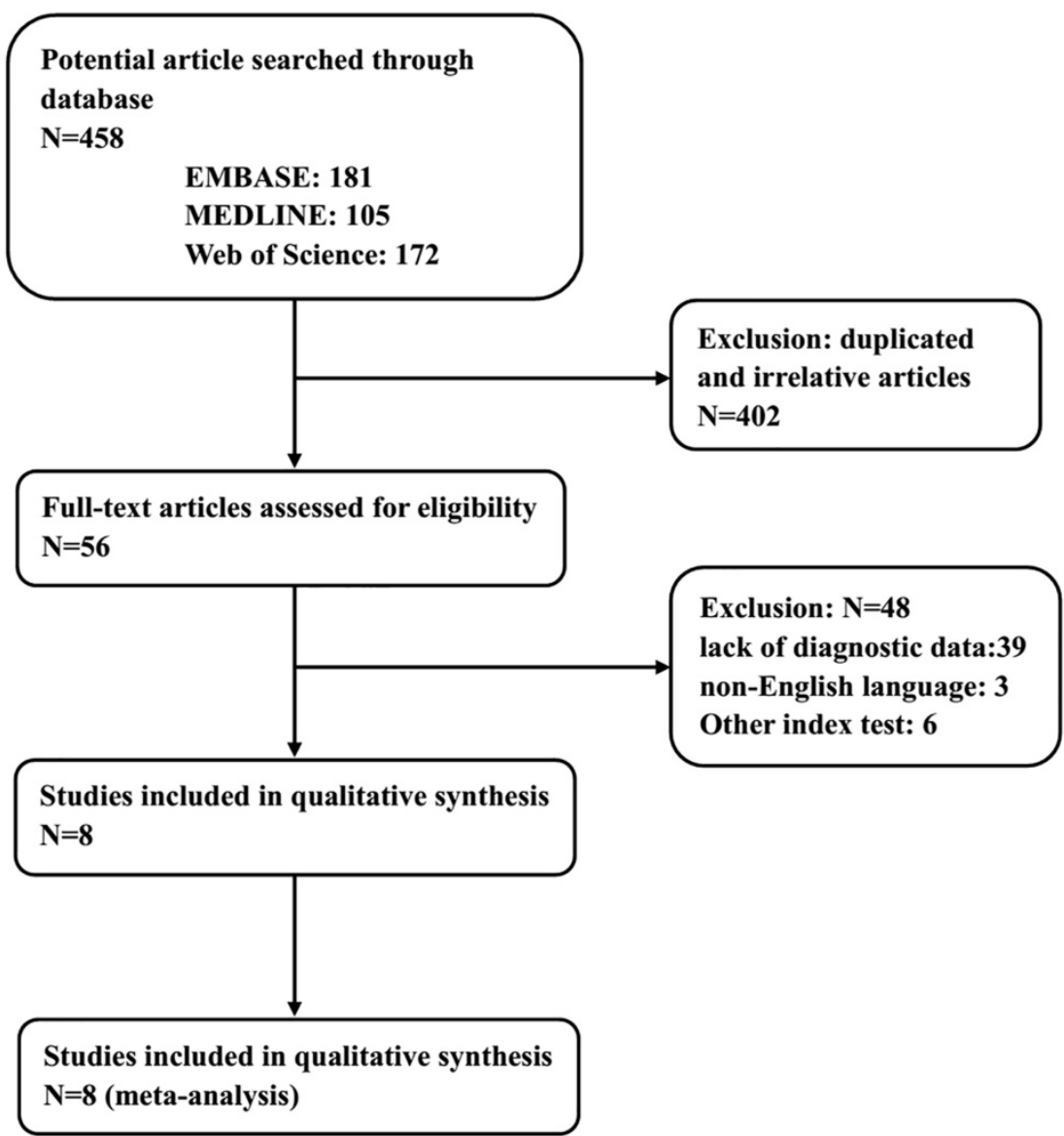

Figure 1. Flow chart showing the study selection process. 
Table 1. Characteristics of the Included Studies

\begin{tabular}{|c|c|c|c|c|c|c|c|c|c|c|}
\hline Author & Year & $\begin{array}{l}\text { Origin of } \\
\text { patients }\end{array}$ & $\begin{array}{l}\text { NO. of } \\
\text { sample }\end{array}$ & $\begin{array}{l}\text { Sample } \\
\text { origin }\end{array}$ & $\begin{array}{l}\mathrm{TP} \\
(\%)\end{array}$ & $\begin{array}{l}\text { FP } \\
(\%)\end{array}$ & $\begin{array}{l}\text { FN } \\
(\%)\end{array}$ & $\begin{array}{l}\mathrm{TN} \\
(\%)\end{array}$ & $\begin{array}{l}\text { CA125 } \\
\text { Cut-off }\end{array}$ & $\begin{array}{l}\text { diagnosis } \\
\text { biomarker }\end{array}$ \\
\hline Haglund et al & 1986 & Finland & 201 & Serum & 74 & 24 & 21 & 82 & - & CA19-9 \\
\hline Haglund et al & 1986 & Finland & 201 & Serum & 43 & 26 & 52 & 80 & 35 & CA125 \\
\hline Haglund et al & 1986 & Finland & 201 & Serum & 39 & 7 & 56 & 99 & 35 & CA125,CA19-9 \\
\hline Haglund et al & 1986 & Finland & 201 & Serum & 34 & 7 & 53 & 99 & 35 & CA125, CEA \\
\hline Haglund et al & 1986 & Finland & 201 & Serum & 31 & 1 & 56 & 105 & 35 & CA125,CEA,CA19-9 \\
\hline Sakamoto et al & 1987 & Japan & 61 & Serum & 26 & 2 & 4 & 29 & - & CA19-9 \\
\hline Sakamoto et al & 1987 & Japan & 61 & Serum & 19 & 1 & 11 & 30 & 32 & CA125 \\
\hline Sakamoto et al & 1987 & Japan & 61 & Serum & 26 & 8 & 4 & 23 & 32 & CA125,CEA \\
\hline Sakamoto et al & 1987 & Japan & 61 & Serum & 29 & 2 & 1 & 29 & 32 & CA125,CA19-9 \\
\hline Benini et al & 1988 & America & 68 & Serum & 14 & 6 & 11 & 37 & - & CA19-9 \\
\hline Benini et al & 1988 & America & 68 & Serum & 14 & 6 & 11 & 37 & 65 & CA125 \\
\hline Benini et al & 1988 & America & 193 & Serum & 19 & 42 & 6 & 126 & 65 & CA125,CEA \\
\hline Benini et al & 1988 & America & 193 & Serum & 24 & 40 & 6 & 128 & 65 & CA125,CA19-9 \\
\hline C wik et al & 2006 & Poland & 110 & Serum & 59 & 4 & 15 & 32 & - & CA19-9 \\
\hline C wik et al & 2006 & Poland & 110 & Serum & 45 & 6 & 29 & 30 & 35 & CA125 \\
\hline$C$ wik et al & 2006 & Poland & 110 & Serum & 39 & 2 & 35 & 34 & 35 & CA125,CA19-9 \\
\hline Duraker et al & 2007 & Turkey & 181 & Serum & 100 & 14 & 23 & 44 & - & CA19-9 \\
\hline Duraker et al & 2007 & Turkey & 181 & Serum & 70 & 13 & 53 & 45 & 35 & CA125 \\
\hline Duraker et al & 2007 & Turkey & 181 & Serum & 38 & 1 & 85 & 57 & 35 & CA125,CEA \\
\hline Duraker et al & 2007 & Turkey & 181 & Serum & 57 & 4 & 66 & 54 & 35 & CA125,CA19-9 \\
\hline Duraker et al & 2007 & Turkey & 181 & Serum & 33 & 1 & 90 & 57 & 35 & CA125,CEA,CA19-9 \\
\hline Luo et al & 2013 & China & 212 & Serum & 107 & 22 & 29 & 54 & 19.7 & CA125 \\
\hline Wang et al & 2013 & China & 145 & Serum & 55 & 10 & 20 & 60 & - & CA19-9 \\
\hline Wang et al & 2013 & China & 145 & Serum & 23 & 8 & 52 & 62 & 33 & CA125 \\
\hline Gu et al & 2015 & China & 132 & Serum & 43 & 33 & 9 & 47 & - & CA19-9 \\
\hline Gu et al & 2015 & China & 132 & Serum & 36 & 26 & 16 & 54 & 35 & CA125 \\
\hline Gu et al & 2015 & China & 132 & Serum & 47 & 5 & 5 & 75 & 35 & CA125,CEA,CA19-9,CA242 \\
\hline
\end{tabular}

$\mathrm{TP}$, true positive rate; $\mathrm{FP}$, false positive rate; $\mathrm{TN}$, true negative rate; $\mathrm{FN}$, false negative rate

\section{QUADAS-2 assessment}

The authors' judgment on risk of bias was determined using the revised assessment tool QUADAS-2. The overall methodological quality of the eligible studies is shown in Figure 2. In the area of patient selection, four studies were judged as having a low risk of bias, two were high risk due to the case-control design [34,36], and the remaining two were unclear $[8,35]$. Three studies were deemed to have a high risk of bias in the conduct of the index test, as the index test was performed after the reference standard in these studies [8, 33, 35]. All studies had a low risk of bias attributed to the reference standard, as this was deemed an objective histological assessment. Five of the 8 studies had a low risk of bias in patient timing and flow. Two studies were described as having a high risk of bias in this area, as not all of the patients who were recruited for these studies could be included in the analysis [7, 35]. Regarding applicability concerns, four studies were low risk for patient selection, and four studies were unclear due to the case-control design and other reasons [8, 34-36]. All studies were considered to have low risk in the applicability of the index test and reference standard.

\section{Diagnostic accuracy assessment and clinical value}

The variations in sensitivity, specificity, PLR and NLR between individual studies of the detection of pancreatic cancer by serum biomarker measurements were illustrated by forest plots. Eight studies including 1235 participants (602 with pancreatic cancer, 633 controls) were included in this meta-analysis. The pooling accuracy analysis of CA125 alone indicated that the pooled sensitivity was 0.59 (95\% CI: $0.54-0.62)$, the specificity was 0.78 (95\% CI: 0.75-0.82), the PLR was 2.57 (95\% CI: 2.03-3.25), and the NLR was $0.52 \quad(95 \%$ CI: $0.40-0.66)$ (Supplementary Figure S1). Similarly, a total of 12 groups tested with a CA125-based panel in all eligible studies were also examined in this meta-analysis. The serum CA125-based panel had a pooled sensitivity of 0.47 (95\% CI $0.47-0.51)$, a specificity of 0.88 (95\% CI 0.86-0.90), a PLR of 6.83 (95\% CI: 4.18-11.13), and an NLR of 0.50 (95\% CI: 0.40-0.62) (Supplementary Figure S2). In addition, the pooling accuracy of CA19-9 alone was also determined in this meta-analysis (Supplementary Figure S3). The pooled results are listed in Table 2 . The heterogeneity of these studies was tested by the I-square values of sensitivity, specificity, PLR, NLR and DOR. From the results, we can see that there was significant heterogeneity between these studies, so the pooled 
indicators were calculated using the random effect model.

The summary receiver operating characteristic (SROC) curve for the tumor diagnostic indicators was drawn based on true-positive rates (sensitivity) for the vertical axis and false-positive rates (1-specifity) for the horizontal axis. An AUC close to 1 reflected a well-performing diagnostic indicator, and poor performance is indicated by an AUC close to 0.5 [37]. As an overall diagnostic indicator, the Q-value is the intersection of the SROC curve with a diagonal line of the ROC diagram from the left upper corner to the right lower corner, reflecting the highest diagnostic value of both sensitivity and specificity. In this meta-analysis, the AUC and Q-value of the CA125-based diagnostic panel were 0.89 and 0.82 , respectively (Figure 3), which is higher than that of CA19-9 alone or CA125 alone (Supplementary Figure S4).

Table 2. Pooled diagnostic accuracy

\begin{tabular}{|c|c|c|c|c|c|c|c|}
\hline & $\begin{array}{l}\text { Diagnostic } \\
\text { biomarker }\end{array}$ & $\begin{array}{l}\text { Sensitivity } \\
(95 \% \mathrm{CI})\end{array}$ & $\begin{array}{l}\text { Specificity } \\
(95 \% \mathrm{CI})\end{array}$ & $\begin{array}{l}\text { PLR } \\
(95 \% \mathrm{CI})\end{array}$ & $\begin{array}{l}\text { NLR } \\
(95 \% \mathrm{CI})\end{array}$ & $\begin{array}{l}\text { DOR } \\
(95 \% \mathrm{CI})\end{array}$ & $\begin{array}{l}\text { AUC } \\
\text { (SEM) }\end{array}$ \\
\hline \multirow[t]{3}{*}{ PC vs non-PC } & CA19-9 & $0.78(0.74-0.82)$ & $0.78(0.74-0.82)$ & $3.95(2.60-6.00)$ & $0.29(0.23-0.36)$ & $14.09(8.84-22.45)$ & 0.85 \\
\hline & CA125 & $0.59(0.54-0.62)$ & $0.78(0.75-0.82)$ & $2.57(2.03-3.25)$ & $0.52(0.40-0.66)$ & $5.45(3.50-8.49)$ & 0.76 \\
\hline & CA125-based panel & $0.47(0.47-0.51)$ & $0.88(0.86-0.90)$ & $6.83(4.18-11.13)$ & $0.50(0.40-0.62)$ & 19.75 (11.35-34.36) & 0.89 \\
\hline \multirow[t]{3}{*}{ Heterogeneity, I2(P) } & CA19-9 & $39.9 \%(0.125)$ & $78.6 \%(0.000)$ & $74.9 \%(0.000)$ & $40.5 \%(0.121)$ & $41.4 \%(0.115)$ & - \\
\hline & CA125 & $88.0 \%(0.000)$ & $70.6 \%(0.001)$ & $32.3 \%(0.170)$ & $81.9 \%(0.000)$ & $53.2 \%(0.036)$ & - \\
\hline & CA125-based panel & $93.4 \%(0.000)$ & $88.4 \%(0.000)$ & $78.5 \%(0.000)$ & $89.6 \%(0.000)$ & $54.7 \%(0.011)$ & - \\
\hline
\end{tabular}

PC, pancreatic cancer; PLR, positive likelihood ratio; NLR, negative likelihood ratio; DOR, diagnostic odds ratio; $A U C$, the area under the curve; $\mathrm{CI}$, confidence interval.

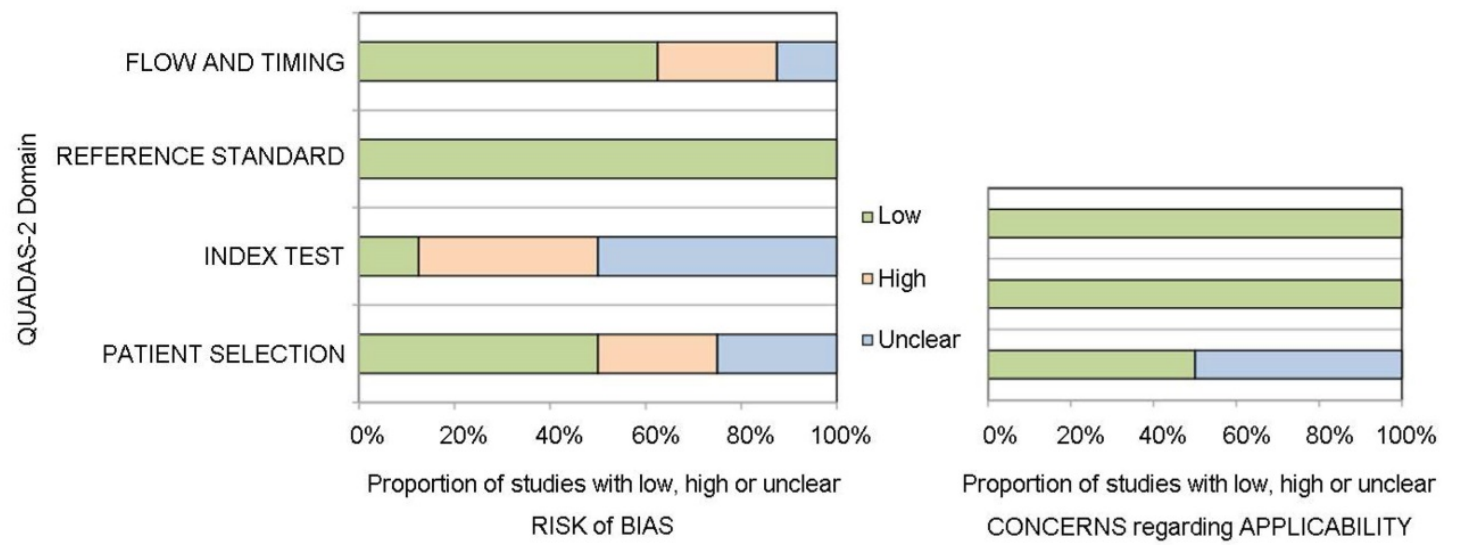

Figure 2. Quality assessment using the QUADAS-2 tool.

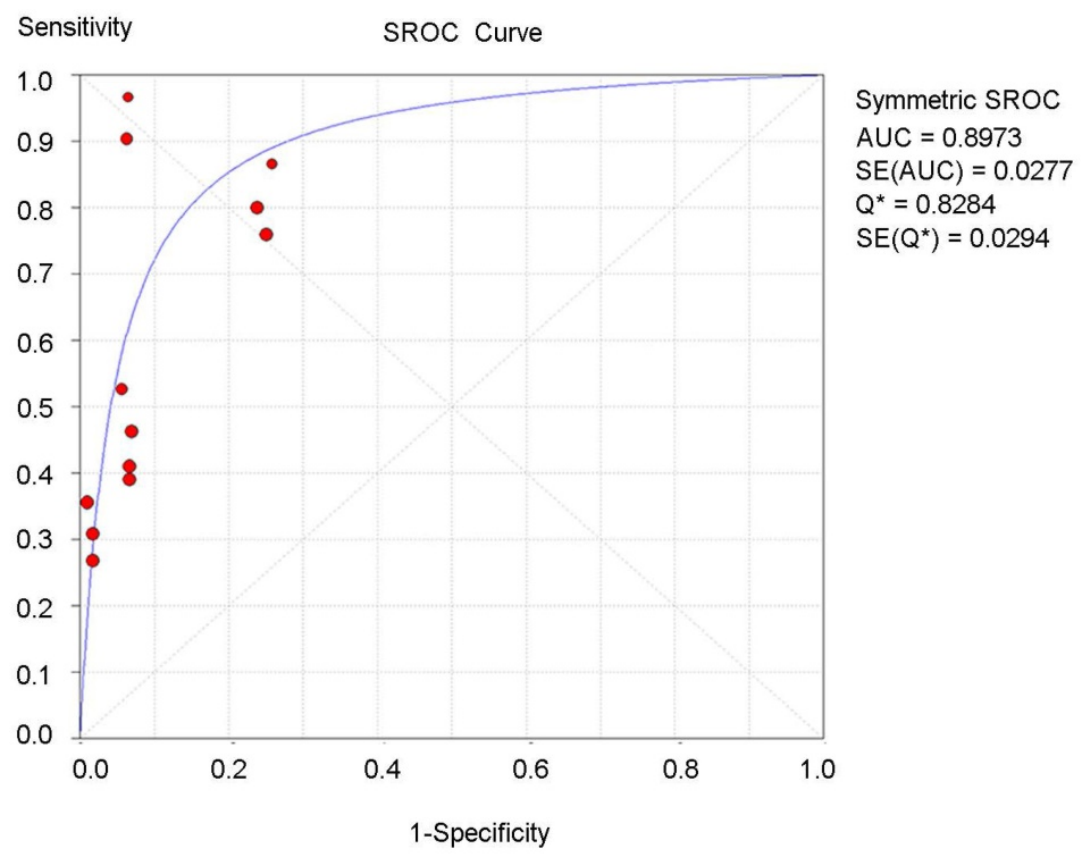

Figure 3. Summary receiver operating characteristic (SROC) curve for the CA125-based biomarker panel in the overall diagnostic accuracy of pancreatic cancer. 


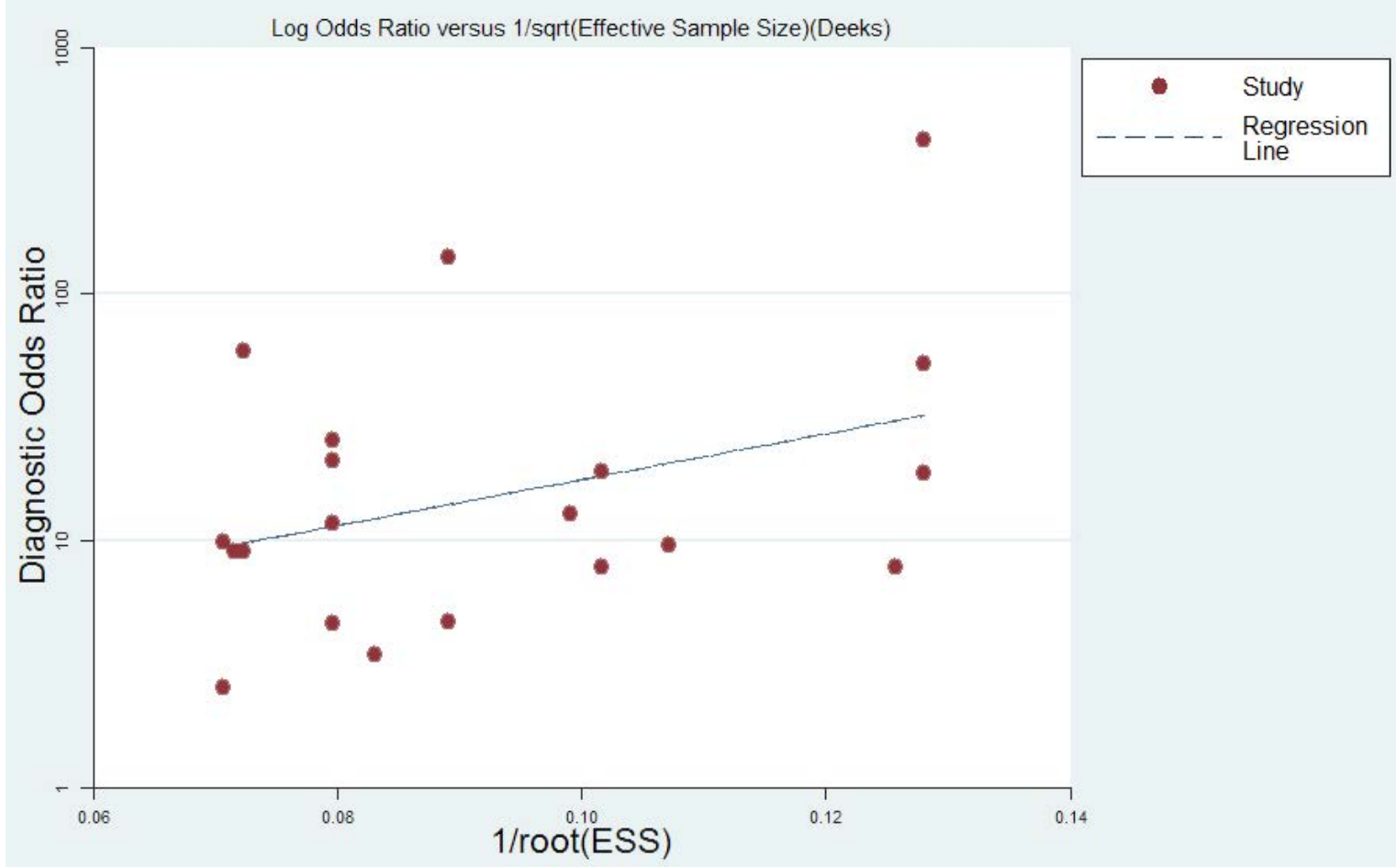

Figure 4. Funnel graph for the assessment of the potential publication bias of the eligible studies.

\section{Publication bias}

The publication bias of the included studies was assessed by funnel plots and Deeks' tests in this meta-analysis. Formal testing for publication bias may be conducted by a regression of the diagnostic log odds ratio (lnDOR) against the square root of the effective sample size (1/ESS1/2), weighting by ESS, with $\mathrm{P}<0.05$ for the slope coefficient indicating significant asymmetry [31]. Publication bias was not found in this meta-analysis according to both the funnel plot, which is almost symmetric, and Deeks' test $(\mathrm{P}=0.164)$. The funnel plot is shown in Figure 4.

\section{Discussion}

The correct and effective diagnosis of pancreatic cancer remains an area of intensive research. Diagnostic limitations still exist in terms of sensitivity, specificity, and cost-effectiveness, although various diagnostic imaging techniques are more or less useful than others. As a relatively simple and reliable noninvasive diagnostic technique, various serum markers of potential value in the diagnosis of pancreatic cancer have been evaluated, especially CA19-9, CA125 and CEA $[25,32]$. Though the CA 19-9 test has certain limitations regarding its sensitivity, as a leading tumor marker, it is still used in the diagnosis of pancreatic carcinoma owing to its high specificity. In pancreatic cancer, many studies have investigated the diagnostic value of CA125, though some have used small sample sizes and retrospective reports. In addition, no meta-analyses have formerly been performed on the diagnostic significance of CA125 in pancreatic cancer. To clarify the clinical value of CA125 and explore its role in the diagnosis of pancreatic cancer, we carried out a meta-analysis.

In the current meta-analysis, which pooled global high-quality studies concerning biomarkers and pancreatic cancer diagnosis, it was demonstrated that the specificity of CA125-based diagnostic panels including CA125/CEA, CA125/CA19-9 and CA125/CEA/CA19-9 was superior to CA125 or CA19-9 alone. Nevertheless, these panels also have some limitations in terms of their sensitivity. As an evaluation index of the overall performance of diagnostic tests, the DOR has significant advantages, in that it is not affected by the morbidity and takes into account the sensitivity and specificity. The pooled DOR of CA125-based diagnostic panels was 19.75, indicating a higher diagnostic accuracy. Similarly, the AUC is also a very important indicator of diagnostic accuracy, similar to the DOR. In the current meta-analysis, the AUC of the CA19-9 test was 0.85. This result was similar to those described by others, where the AUC of the CA19-9 test ranged between $72 \%$ and $87 \%$ [38-40]. The AUC of the CA125-based diagnostic panel was 0.89 , which demonstrates a better diagnostic capability compared with CA125 or CA19-9 alone. Hence, these indicators reflect the promising clinical value of the CA125-based 
diagnostic panel as a diagnostic biomarker.

CA125, which is encoded by the MUC16 gene, is primarily known as a useful serological marker for the clinical management of ovarian cancer [41]. However, an increasing number of studies have proposed a role for serum CA125 as a marker for pancreatic carcinoma diagnosis. More importantly, since serum levels of CA125 are not correlated with serum bilirubin levels, its positivity is not significantly different between the jaundiced and non-jaundiced subgroups of patients with both pancreatic carcinoma and benign pancreatic diseases [8, 42], which is superior to the serum CA19-9 test. Hence, CA125 can be useful in the diagnosis of pancreatic cancer, especially in jaundiced patients.

Furthermore, it was demonstrated that CA125 could also play an important role in predicting survival in these patients. In this field, our team has previously performed a series of relevant studies [24-26, 42]. A CEA+/CA125+/CA19-9 >1000 U/mL serum signature was identified as a preoperative indicator for surgical outcome in pancreatic cancer, which implied that patients with this index hardly benefited from pancreatectomy, even if the resection was successful with an R0-definition [24, 26]. Abnormally high levels of CA125 were observed in some patients who underwent pancreatectomy and experienced early postoperative metastasis at distant organs, which may indicate that CA125 is a valuable clinical biomarker of occult disease in pancreatic cancer [24, 25]. In addition, an elevated preoperative CA125 level was an independent prognostic factor for pancreatic cancer patients with non-elevated bilirubin or hyperbilirubinemia [42]. These theories support the clinical value of the serum CA125 level in predicting pancreatic cancer.

Several limitations should be considered in the current meta-analysis. First, the sample size was still small. Though we pooled all participants in the eligible studies, some were excluded due to the involvement of other gastrointestinal cancers, and only 1235 participants were studied in the analysis of overall diagnosis. Second, the heterogeneity of the population was increased due to differences in patient characteristics (age, race, country, etc.) and cut-off criteria. Third, only studies published in English were included in our meta-analysis, which may also result in bias. Finally, in order to further validate the CA125-based diagnostic panel in the diagnosis of pancreatic cancer, we need more multi-center, prospective clinical studies. Thus, our results might be flawed to some extent.

\section{Conclusions}

In summary, the present meta-analysis, representing a quantified synthesis of the published studies, has shown that a CA125-based diagnostic panel is better at diagnosing pancreatic cancer than testing CA125 or CA19-9 alone. As it is the most economic, convenient, safe and rapid method, the CA125-based diagnostic panel should be widely used to complement other diagnostic techniques. However, further studies are required to confirm our conclusion.

\section{Supplementary Material}

Supplementary figures.

http://www.jcancer.org/v08p3615s1.pdf

\section{Acknowledgements}

This study was jointly funded by the National Science Fund for Distinguished Young Scholars of China (NO.81625016), the National Natural Science Foundation of China (NO.81372651, 81502031 and $81602085)$ and the Shanghai Sailing Program (17YF1402500).

\section{Contribution to authorship}

Conceived and designed the experiments: XJY, QCM, SS \& CL. Performed the experiments: QCM, JFX, DKL \& BZ. Analyzed the data: SS, YQ \& SRJ. Contributed reagents/materials/analysis tools: CL, WYX, JX \& QXN. Wrote the paper: QCM.

\section{Competing Interests}

The authors have declared that no competing interest exists.

\section{References}

1. Ferlay J, Steliarova-Foucher E, Lortet-Tieulent J, et al. Cancer incidence and mortality patterns in Europe: estimates for 40 countries in 2012. Eur J Cancer. 2013; 49: 1374-403.

2. Hidalgo M. Pancreatic cancer. N Engl J Med. 2010; 362: 1605-17.

3. Siegel RL, Miller KD, Jemal A. Cancer statistics, 2016. CA Cancer J Clin. 2016; 66: 7-30.

4. Rosch T, Dittler HJ, Strobel K, et al. Endoscopic ultrasound criteria for vascular invasion in the staging of cancer of the head of the pancreas: a blind reevaluation of videotapes. Gastrointest Endosc. 2000; 52: 469-77.

5. Vargas R, Nino-Murcia M, Trueblood W, et al. MDCT in Pancreatic adenocarcinoma: prediction of vascular invasion and resectability using a multiphasic technique with curved planar reformations. AJR Am J Roentgenol. 2004; 182: 419-25.

6. Bunger S, Laubert T, Roblick UJ, et al. Serum biomarkers for improved diagnostic of pancreatic cancer: a current overview. J Cancer Res Clin Oncol. 2011; 137: 375-89.

7. Duraker N, Hot S, Polat $Y$, et al. CEA, CA 19-9, and CA 125 in the differential diagnosis of benign and malignant pancreatic diseases with or without jaundice. J Surg Oncol. 2007; 95: 142-7.

8. Haglund C. Tumour marker antigen CA125 in pancreatic cancer: a comparison with CA19-9 and CEA. Br J Cancer. 1986; 54: 897-901.

9. Safi F, Roscher R, Beger HG. Tumor markers in pancreatic cancer. Sensitivity and specificity of CA 19-9. Hepatogastroenterology. 1989; 36: 419-23.

10. Steinberg WM, Gelfand R, Anderson KK, et al. Comparison of the sensitivity and specificity of the CA19-9 and carcinoembryonic antigen assays in detecting cancer of the pancreas. Gastroenterology. 1986; 90: 343-9.

11. Tempero MA, Uchida E, Takasaki $\mathrm{H}$, et al. Relationship of carbohydrate antigen 19-9 and Lewis antigens in pancreatic cancer. Cancer Res. 1987; 47: 5501-3.

12. Ferrone CR, Finkelstein DM, Thayer SP, et al. Perioperative CA19-9 levels can predict stage and survival in patients with resectable pancreatic adenocarcinoma. J Clin Oncol. 2006; 24: 2897-902. 
13. Hata S, Sakamoto Y, Yamamoto Y, et al. Prognostic impact of postoperative serum CA 19-9 levels in patients with resectable pancreatic cancer. Ann Surg Oncol. 2012; 19: 636-41.

14. Tempero MA, Arnoletti JP, Behrman S, et al. Pancreatic adenocarcinoma. J Natl Compr Canc Netw. 2010; 8: 972-1017.

15. Jacobs I. Screening for ovarian cancer by CA-125 measurement. Lancet. 1988; 1: 889.

16. Giessen C, Nagel D, Glas M, et al. Evaluation of preoperative serum markers for individual patient prognosis in stage I-III rectal cancer. Tumour Biol. 2014; 35: 10237-48.

17. Shia J, Agaram NP, Olgac S, et al. Adenocarcinoma of the minor duodenal papilla and its precursor lesions: a clinical and pathologic study. Am J Surg Pathol. 2014; 38: 526-33.

18. Wu WR, Shi $\mathrm{XD}$, Zhang $\mathrm{R}$, et al. Clinicopathological significance of aberrant Notch receptors in intrahepatic cholangiocarcinoma. Int J Clin Exp Pathol. 2014; 7: 3272-9.

19. Chakraborty S, Baine MJ, Sasson AR, et al. Current status of molecular markers for early detection of sporadic pancreatic cancer. Biochim Biophys Acta. 2011; 1815: 44-64.

20. Haridas D, Chakraborty S, Ponnusamy MP, et al. Pathobiological implications of MUC16 expression in pancreatic cancer. PloS one. 2011: 6: e26839.

21. Horn A, Chakraborty S, Dey P, et al. Immunocytochemistry for MUC4 and MUC16 is a useful adjunct in the diagnosis of pancreatic adenocarcinoma on fine-needle aspiration cytology. Arch Pathol Lab Med. 2013; 137: 546-51.

22. Shimizu A, Hirono S, Tani M, et al. Coexpression of MUC16 and mesothelin is related to the invasion process in pancreatic ductal adenocarcinoma. Cancer Sci. 2012; 103: 739-46.

23. Streppel MM, Vincent A, Mukherjee R, et al. Mucin 16 (cancer antigen 125) expression in human tissues and cell lines and correlation with clinical outcome in adenocarcinomas of the pancreas, esophagus, stomach, and colon. Hum Pathol. 2012; 43: 1755-63.

24. Luo G, Xiao Z, Long J, et al. CA125 is superior to CA19-9 in predicting the resectability of pancreatic cancer. J Gastrointest Surg. 2013; 17: 2092-8.

25. Liu $\mathrm{L}, \mathrm{Xu} \mathrm{H}$, Wang $\mathrm{W}$, et al. A preoperative serum signature of $\mathrm{CEA}+/ \mathrm{CA} 125+/ \mathrm{CA} 19-9>/=1000 \mathrm{U} / \mathrm{mL}$ indicates poor outcome to pancreatectomy for pancreatic cancer. Int J Cancer. 2015; 136: 2216-27.

26. Liu L, Xu HX, Wang WQ, et al. Serum CA125 is a novel predictive marker for pancreatic cancer metastasis and correlates with the metastasis-associated burden. Oncotarget. 2016; 7: 5943-56.

27. Whiting PF, Rutjes AW, Westwood ME, et al. QUADAS-2: a revised tool for the quality assessment of diagnostic accuracy studies. Ann Intern Med. 2011; 155: 529-36.

28. Knobloch K, Yoon U, Vogt PM. Preferred reporting items for systematic reviews and meta-analyses (PRISMA) statement and publication bias. $J$ Craniomaxillofac Surg. 2011; 39: 91-2.

29. Walter SD. Properties of the summary receiver operating characteristic (SROC) curve for diagnostic test data. Stat Med. 2002; 21: 1237-56.

30. DerSimonian R, Laird N. Meta-analysis in clinical trials revisited. Contemp Clin Trials. 2015; 45: 139-45.

31. Deeks JJ, Macaskill P, Irwig L. The performance of tests of publication bias and other sample size effects in systematic reviews of diagnostic test accuracy was assessed. J Clin Epidemiol. 2005; 58: 882-93.

32. Benini L, Cavallini G, Zordan D, et al. A clinical evaluation of monoclonal (CA19-9, CA50, CA12-5) and polyclonal (CEA, TPA) antibody-defined antigens for the diagnosis of pancreatic cancer. Pancreas. 1988; 3: 61-6.

33. Cwik G, Wallner G, Skoczylas T, et al. Cancer antigens 19-9 and 125 in the differential diagnosis of pancreatic mass lesions. Arch Surg. 2006; 141: 968-73.

34. Gu YL, Lan C, Pei H, et al. Applicative Value of Serum CA19-9, CEA, CA125 and CA242 in Diagnosis and Prognosis for Patients with Pancreatic Cancer Treated by Concurrent Chemoradiotherapy. Asian Pac J Cancer Prev. 2015; 16: 6569-73.

35. Sakamoto $\mathrm{K}$, Haga $\mathrm{Y}$, Yoshimura R, et al. Comparative effectiveness of the tumour diagnostics, CA 19-9, CA 125 and carcinoembryonic antigen in patients with diseases of the digestive system. Gut. 1987; 28: 323-9.

36. Wang Z, Tian YP. Clinical value of serum tumor markers CA19-9, CA125 and CA72-4 in the diagnosis of pancreatic carcinoma. Mol Clin Oncol. 2014; 2: 265-8.

37. Edge SB, Compton CC. The American Joint Committee on Cancer: the 7th edition of the AJCC cancer staging manual and the future of TNM. Ann Surg Oncol. 2010;17:1471-4.

38. Chan A, Prassas I, Dimitromanolakis A, et al. Validation of biomarkers that complement CA19.9 in detecting early pancreatic cancer. Clin Cancer Res. 2014; 20: 5787-95.

39. Huang Z, Liu F. Diagnostic value of serum carbohydrate antigen 19-9 in pancreatic cancer: a meta-analysis. Tumour Biol. 2014; 35: 7459-65.

40. Kim YC, Kim HJ, Park JH, et al. Can preoperative CA19-9 and CEA levels predict the resectability of patients with pancreatic adenocarcinoma? I Gastroenterol Hepatol. 2009; 24: 1869-75.

41. Yin BW, Lloyd KO. Molecular cloning of the CA125 ovarian cancer antigen: identification as a new mucin, MUC16. J Biol Chem. 2001; 276: 27371-5.

42. Chen T, Zhang MG, Xu HX, et al. Preoperative serum CA125 levels predict the prognosis in hyperbilirubinemia patients with resectable pancreatic ductal adenocarcinoma. Medicine. 2015; 94: e751. 